\title{
PERSPECTIVAS POLITICAS, SOCIALES Y ETICAS DE LA INVESTIGACION EN UNA ERA DE BARBARIE*
}

Jaime Breilh**

Breilh J. Perspectivas politicas, sociales y eticas de la investigacion en una era de barbarie. Rev Esc Enferm USP 2002; 36(3): 210-21.

Las ramas profesionales como la enfermería que se desarrollan cerca de la necesidad humana, pueden construir una actitud más solidaria $y$ democrática en la investigación.

No nos sorprende, entonces, que las organizaciones de enfermería del Brasil -país que lleva una delantera en América Latina en la institucionalización de una salud colectiva progresista-, convoquen a este nuevo encuentro internacional para avalizar la trayectoria de la investigación y resalten la urgencia de estudiar las perspectivas politicas, sociales y éticas de la investigación, justamente en momentos en que se reconoce una crisis del modelo científico dominante.

Frente a este llamado de reflexión tenemos que enfocar aquellos temas que nos permitan responder a ias lacerantes urgencias de una época que con mucha razón ha sido caracterizada como una nueva era de barbarie o capitalismo salvaje.

Ahora bien, poco conseguiriamos si nos limitamos a trazar aqui algunos rasgos de la tragedia actual de América Latina y elaboramos un inventario de recetas sobre la investigación debida. De ese modo, no quedaria explicada ni la crisis social, ni la crisis actual de la investigación, ni tampoco la relación entre una y otra. Por eso hemos orientado estas reflexiones para trascender lo descriptivo y articular el análisis de ias dos crisis. Un enfoque que nos permita mirar la investigación de las condiciones de salud en una era de tragedia social como principal objeto, $y$ que a la vez nos empuje, desde los nuevos horizontes de la epistemologia, a mirar también las paradojas y empobrecimientos que pesan sobre el sujeto del conocimiento. En definitiva, asumimos que el desafio mayor de este evento es reconocer las claves para conformar un paradigma emancipador de la ciencia en salud.

\section{ALGUNAS CLAVES EPISTEMOLÓGICAS: UNA NECESARIA RELECTURA DE KHUN}

En mi libro la "Epidemiología Crítica" que se publicará próximamente en Buenos Aires(2), dedico un capítulo entero al análisis de "cómo se transforman, avanzan o retroceden los conceptos científicos", y pongo de relieve la necesidad de estudiar con precaución el torrente de materiales que ha producido la llamada "epistemología posmoderna". Nos motivó la preocupación de cuestionar los modelos descriptivos (positivistas y racionalistas) de la investigación de la modernidad, pero también de alertar sobre el crecimiento actual de una renovada ideologia conservadora que se presenta con el membrete de "posnormal" o "posmoderna".

Nuestra posición en ese debate - $y$ que no podemos ampliaria en estas páginas-, es la de que la construcción de una ciencia contrahegemónica pasa necesariamente por el análisis de los aportes actuales que desde la epistemología, desde las ciencias humanas, o aun desde el pensamiento de las organizaciones sociales, contribuyen a reconstruir tanto el objeto de estudio como el sujeto del conocimiento, y que implican una enriquecedora y selectiva recuperación de ideas vitales de la filosofia revolucionaria de los dos siglos anteriores. Hacerlo, por ejemplo, con trabajos como los de Edgar Morin( ${ }^{2)}$, que a la par que condenan el distanciamiento de la ciencia respecto a la realidad, trabajan también en el problema de la construcción del objeto de la ciencia, recuperando la noción de complejidad que se extravió bajo la mirada lineal y reduccionista del positivismo y evitando el uso de megarelatos impositivos que han reducido el pensamiento científico al molde de una visión rígida y monótona de la realidad; también planteamientos como los de Boaventura Santos (3) , con su idea de una segunda ruptura epistemológica que nos acerque al saber popular; o ideas como las de Maturana contra esa noción de "objetividad que obliga"; o aquellas de Bruno Latour (4) con su cuestionamiento a la idea de un mundo ficticiamente exterior, provocado por el divorcio metodológico entre objeto y sujeto, que en el positivismo constituye un obstáculo para la objetividad; o planteamientos como los de McLaren (5) y Walsh et al (6), con sus propuestas de descolonización e indisciplinamiento de la ciencia para incorporar el multiculturalismo en ella.

\footnotetext{
* Conferência apresentada no II Encontro Internacional de Pesquisa em Enfermagem: trajetória espaço-temporal da Pesquisa

** Jaime Breilh, Md.PhD. Director del CEAS (Centro de Estudios y Asesoría en Salud); Presidente del CINDES; Asturias N2402 (La Floresta-Quito, Ecuador) jbreilh ceas.med.ec
} 
En fin, contribuciones éstas que implican un notable trabajo de reflexión, que se deben complementar con los innovadores aportes surgidos desde las ciencias sociales, donde descollan algunos científicos latinoamericanos, o con aquellos surgidos desde la lucha social y los expertos del pueblo, cuyas ideas también están contribuyendo a conocer mejor las raíces de la crisis actual del capitalismo, y a perfilar ese "atro mundo posible" del que tanto depende la salud en el mundo.

De hecho, nosotros hemos realizado un estudio de ese tipo de ideas renovadoras, todas las cuales trabajan en dirección de superar la linealidad del pensamiento cientifico dominante, con su basamento empírico descriptivo y su construcción eurocéntrica. La misma producción reciente de la salud colectiva latinoamericana, en la cual ocupan un sitial de relieve varios autores del Brasil, ofrece contribuciones importantes que han refrescado la epistemologia en salud. Entonces, reconocemos, sin duda, la necesidad de este movimiento de renovación del conocimiento, del cual hemos sido parte, y que algunos prefieren designarlo con el polémico nombre de ciencia "posnormal" o "posmoderna", pero, a la vez, enfatizamos en la necesidad de hacerlo en el marco de esa filosofia crítica de la ciencia, que se consolidó desde los revolucionarios alemanes del Siglo XIX y que sigue reclamando ahora que trabajemos para conocer la diversidad, lo micro, el mundo individual, pero sin perder la unidad, la noción de totalidad que nos une bajo una estructura de profunda inequidad social, ni peor caer en deconstrucciones que nos devuelvan a una visión fragmentada del objeto y a una atomización del sujeto. Como diria Best(7), no se trata de sustituir "la tirania de la totalidad" por la "dictadura del fragmento".

Hemos sostenido además, que ante la crisis del pensamiento científico moderno es importante una relectura del texto clássico de Thomas Khun sobre "La Estructura de las Revoluciones Científicas"(8) para comprender y orientar los cambios acelerados del modelo de conocimiento.

El modelo de Khun, puso de manifiesto la noción de transformabilidad y movimiento de la ciencia, resaltando el carácter contradictorio de esa transformación y el peso de las contradicciones sociales y de intereses sobre los aspectos "normales" y alternativos del quehacer científico. Reconoció, en definitiva, el carácter sociológico o determinación "comunitaria" de la ciencia, sus modelos e instrumentos, devolviendo a la cienciología una perspectiva social que se había perdido en los abordajes historiográficos liberales o personalistas.

Es verdad que se han detectado inconsistencias en el planteamiento kuhniano original, por que en la explicación de las crisis de la ciencia solo tomb encuenta la incapacidad del viejo paradigma para explicar "anomalias", sin atender la existencia de "paradojas", "rebasamientos de limites" y "puntos ciegos" o invisibilización de ciertas temáticas(9)

Nosotros hemos advertido además, que para explicar una crisis del paradigma imperante en un campo científico no deben solamente enfocarse los obstáculos originados en aquellas anomalias, paradojas o puntos ciegos, detectados por una comunidad científica, sino aquellos que resultan de las contradicciones más amplias de la sociedad, la lucha de intereses y visiones que operan en ella $y$ presionan para el cambio de los paradigmas; muchas veces en direcciones diferentes a las que se esperaria según la lógica interna de un campo científico, y muchas otras, sin mediar siquiera un proceso importante de acumulación de anomalias, ni rebasamientos, ni paradojas. Pero dichas falencias no deben hacernos perder de vista la riqueza del análisis integrado que propuso Thomas Khun.

Con esta defensa del espíritu del enfoque de Kuhn y la recomendación de contextualizar el análisis de las tendencias científicas -incorporando las determinaciones económicas, politicas y culturales más amplias-, no pretendemos inducir a un enfoque determinista y mecánico, que desconozca la importancia de los hechos y relaciones particulares que ocurren al interior de la comunidad de "especialistas"; más bien, lo que reclamamos es entender la medida en que cada una de esas dimensiones, macro y micro, incide en la determinación de las proyecciones politicas, sociales y éticas de la investigación. Estas últimas, las que pertenecen al mundo micro en que trabajan los especialistas, permitiendo la génesis de productos e ideas específicas, y las primeras, que corresponden a la dimensión macro de las relaciones históricas sociales, modelando la reproducción social del conjunto y los modos de vida en cuyo seno se establecen las posibilidades y limites del quehacer de la ciencia.

Ningún discurso científico se genera al margen de una práctica social, y tanto el discurso como la práctica científicos se recrean constantemente en medio de las condiciones de posibilidad de lo que se puede pensar, conocer $y$ decir en un momento histórico determinado. La construcción del discurso científico no puede siquiera deslindarse del discurso social común; pues hasta las estructuras lingüísticas y los sentidos y significaciones que subyacen en un contexto cultural inciden sobre la producción de ideas cientificas .

El análisis del proceso de producción de conocimientos científicos, entonces, "no se refiere sólo a las determinaciones en el modo de pensar cientificamente de los/as expertos/as -descubrir y validar- sino que debe enfocar las determinaciones estructurales y político ideológicas de procesos no 
culturales ni psicológicos, como los sistemas de financiamiento y poder que caracterizan los escenarios donde se produce ciencia, y que no sólo tienen impactos sobre individuos y grupos sino sobre la conformación de las condiciones económicas y políticas más amplias que moldean, indirectamente, la episteme y las estructuras simbólicas en cuyo seno se desenvuelve el quehacer. Es decir, tampoco dejan de pesar los procesos de la cultura en la que trabajan las personas que hacen ciencia y las estructuras simbólicas que condicionan, directa o indirectamente, los procesos de interpretación. Por fin, inciden también las relaciones de poder y las tendencias ideológicas de los escenarios educativos, especialmente las universidades, que participan en la reproducción del pensamiento social y técnico de una sociedad. En otras palabras, y por todo lo analizado, no se trata sólo de una psicologia y antropologia del problema, sino de una sociologia y economia política del mismo; no se trata sólo de una psicogenética de la producción de ideas científicas, cuanto de una filosofia critica del problema" . Relaciones que podriamos representar como el movimiento de una historia "externa", con sus mediaciones económicas, institucionales y culturales, $y$ una historia "interna" de los escenarios especializados del quehacer cientifico, mediados a su vez, por el modo de vida posible de las actores involucrados en todo el conjunto.

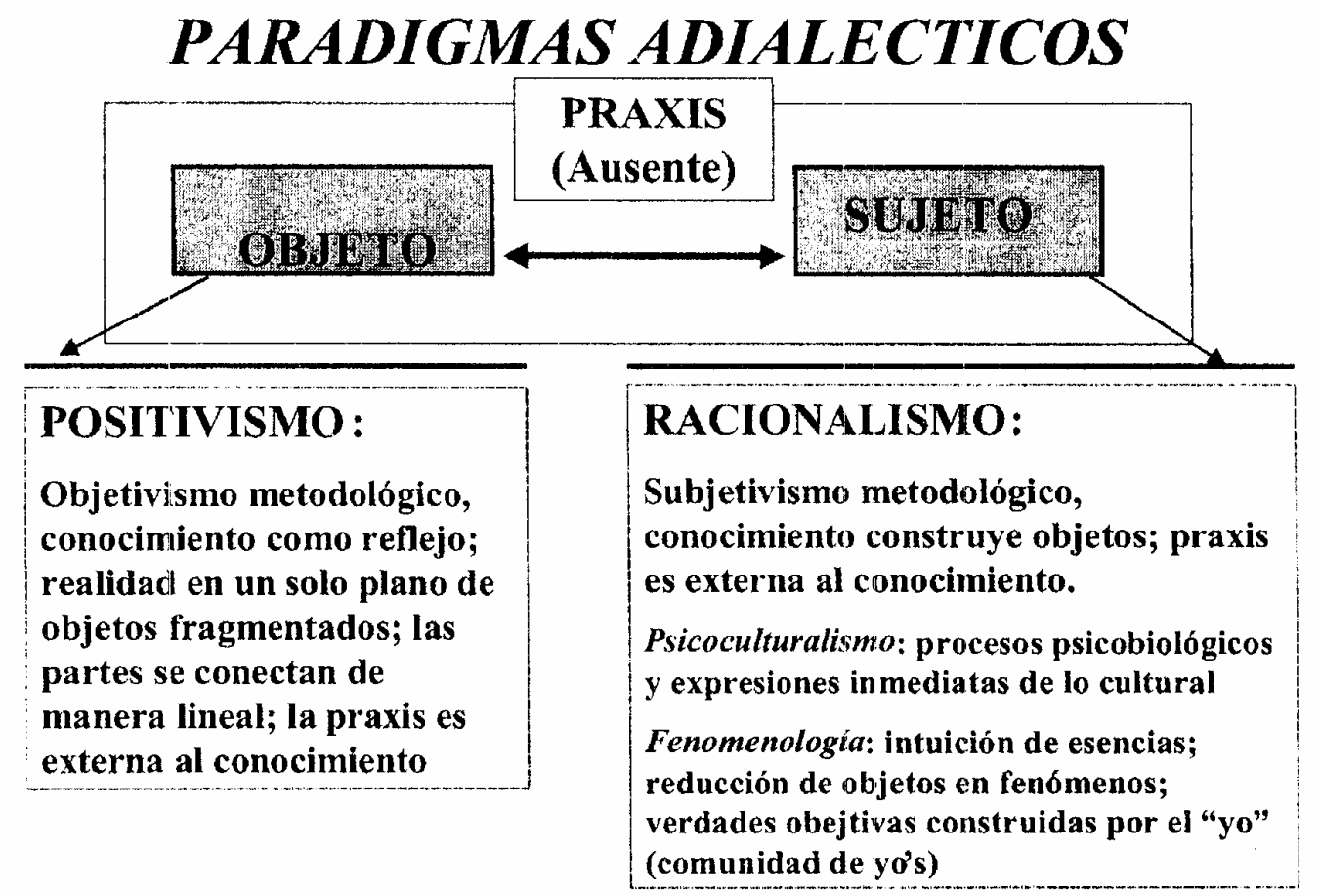

De esa forma, para responder al análisis de las perspectivas políticas, sociales y éticas de la investigación, debemos recrear el análisis kuhniano en el movimiento más amplio de las contradicciones de la sociedad, replanteándolo en una epistemología dialéctica. Lo anterior implica ver a la ciencia como un proceso que mantiene una autonomia relativa, pero que está insertado en la vida colectiva e indisolublemente ligado a ias determinaciones de su estructura de poder.

Por todo lo dicho, debemos estar claros de que . el análisis crítico de la producción de conocimientos no es un proceso puramente académico puesto que para realizarlo tenemos necesariamente que relacionar el desafio epistemológico de construir un nuevo paradigma, con el desafio ético de construir un nuevo modelo de praxis en el seno de un escenario histórico donde opera el choque de intereses sociales opuestos y una estructura de poder y dominación. Es decir, el análisis de las perspectivas de la investigación es un acto de desarrollo cognitivo, pero al mismo tiempo es un desafio ético y político.

La salud pública convencional y funcionalista ha trabajado fundamentalmente sobre la base de dos paradigmas surgidos de la desconexión de objeto y sujeto: el paradigma positivista, para el cual la realidad existe como "objetos puros" que se reflejan en el sujeto; y el racionalismo, para el cual es el "sujeto puro", el que construye los objetos de esa realidad (Figura).

No podemos aqui explayarnos en una crítica de esos paradigmas sólo destacaremos dos aspectos de enorme interés para la evaluación del trabajo científico que realizamos: a) Desde cualquiera de esas dos ópticas predominantes, el objeto salud pierde la unidad dialéctica de sus dimensiones: ontológica, conceptual y práctica. Tanto el positivismo como el racionalismo se basan en la desintegración de la salud como objeto de estudio, la salud como concepto y la salud como un campo de intervención. En otras palabras, sea por la 
vía del objetivismo o por la del subjetivismo, dichos paradigmas parten de la separación objeto/sujeto y la asunción de la práctica como un fenómeno externo ai conocimiento; y b) desde el marco de esos paradigmas se aplica un triple reduccionismo: i) reducir la salud al aspecto "negativo" de la enfermedad y a lo individual; ii) reducir la realidad en salud al plano único de los fenómenos empiricamente observables, sean estos cuantitativos o cualitativos; $y$ iii) atribuir el movimiento de esa realidad a la simplicidad unidimensional de un orden mecánicamente determinado sea por leyes asociativas -como es el caso del positivismo- o por construcciones subjetivas. En una impresionante mayoria de proyectos $y$ tesis de investigación de nuestros países se sigue acríticamente unos de estos dos paradigmas, con sus variantes.

La construcción predominante de la relación: "objeto / concepto I campo" de salud que utiliza la salud pública convencional, y que puede traducirse como el conjunto coherente "factor causal/paradigma de riesgo/acción funcionalista ", no nos permite penetrar en la realidad para apoyar su transformación y, por el contrario, constituye una herramienta de mediatización y hegemonia.

Veamos esta lógica que aqui denunciamos. En efecto, primero la noción de "factor causal" que se utiliza es una forma de segmentar la realidad para oscurecerla; luego el paradigma de riesgo designa a esos factores como "riesgos" $y$ establece que es el riesgo el que une a esos fragmentos; y finalmente, a la noción del objeto como factores, y al concepto de riesgo como explicación se articula la noción de "función social" como naturaleza esencial de la acción en salud. En otras palabras, la lógica funcionalista se construye así: unos factores de riesgo convertidos en variables pasan a integrar un modelo matemático formal, una o varias de esas variables adquieren un "peso" mayor en la explicación de la varianza de $\langle\mathrm{y}\rangle$, convirtiéndose inmediatamente en factores de alto riesgo, que por esa via pasan a ser consideradas "causas" del problema; sobre esa base, toda la lógica de la acción se orienta a modificarias como procesos aislados. Bajo ese tipo de construcción, queda por fuera la acción sobre los modos de la vida social y sobre los procesos generativos, ya que todo el esfuerzo se concentra sobre las variables que son los puntos altos del iceberg; la mirada se enfoca entonces sobre ias manifestaciones visibles, factorizadas, de la realidad empírica. La investigación queda así reducida a un conjunto de procedimientos empíricos o, en el mejor de los casos, a una ciencia descriptiva reducida a la construcción de modelos de asociación y conjunción constante. Modelos muchas vetes muy sofisticados que trabajan con asociaciones reales, puesto que el hecho de que sean fenómenos empiricos no significa que no existan efectivamente. Pero con ese tipo de investigación estamos atados a la cosmética social y al funcionalismo.
En el campo de la salud durante todo este tiempo se ha impuesto aquella lógica, la cual contribuyó a hilvanar una estrategia conveniente al poder basada en: [a] la manipulación del conocimiento como reflejo en ias mentes de élites escogidas; [b] la interpretación de la realidad como un objeto fragmentado y cuyos pedazos convertimos en "variables" que las podemos conectar de manera lineal; y [c] la acción como una actividad focalizada en dichas variables, para provocar apenas cambios de forma parciales. ${ }^{10}$

Podemos aseverar entonces que dicho paradigma científico se liga a una concepción política conservadora. El enfoque positivista, así como otras formas de pensamiento que fragmentan la realidad, han llevado a que el trabajo científico termine convirtiéndose, consciente o inconscientemente, en un instrumento funcional a la estructura de poder. Sólo a las clases dominantes interesa que miremos la realidad exclusivamente por sus partes, y que actuemos sólo sobre dichas partes; no conviene al poder que miremos la totalidad histórica y las raíces de los problemas de la salud, no le conviene a sus intereses, por ejemplo, que comprendamos los vínculos profundos que existen entre el sistema monopólico de propiedad y la distribución de modos $y$ calidades de vida entre los diferentes grupos sociales, étnicos y de género.

Por todo lo dicho anteriormente, tenemos que estar claros de que la triple dimensión de la salud como objeto/concepto/campo no corresponde a tres elementos totalmente separables, que podemos escoger y trabajar por una preferencia, sino que son aspectos que guardan profunda concatenación y conforman un sistema de relaciones, que para ser trabajado desde una perspectiva emancipadora, requiere situarse desde una praxis de transformación concreta $y$ apoyarse en una teoria general crítica.

En síntesis, proponemos los siguientes puntos como base de una discusión de los contenidos y proyecciones de una investigación alternativa en salud: 1) trabajar las relaciones entre la determinación por procesos macro y micro sociales, sin absolutizar la determinación de la totalidad estructural, ni tampoco sobrevalorar el peso del microcosmos individual y cotidiano; 2) incorporar la diversidad, pero sin perder de vista las formas de conexión, sobretodo ligadas a la estructura de propiedad $y$ poder de nuestras sociedades; 3) romper el rígido marco de las certezas y simplificaciones neopositivistas, pero sin perder de vista los momentos de regularidad y la operación de leyes, las que no se reducen a expresar conjunciones constantes como lo hace la "teoria del riesgo".

El avance en esos puntos implica una visión nítida y operacionalizable de la complejidad, para buscar un principio de explicación más rico que el de la simplificación (separación/reducción) -que es el que 
se ha impuesto en la ciencia empírico analítica-, pero bajo un replanteamiento que no caiga ni en la rigidez de ningún determinismo, ni en la ambigüedad y fragmentación insoluble del relativismo y del constructivismo culturalista en la salud; y 4) romper los moldes de una construcción eurocéntrica o unicultural, aspecto que trataremos más adelante.

\section{TRAYECTORIA ESPACIO TEMPORAL DE LA VIDA: UN ESCENARIO DE BARBARIE}

La ciencia se mueve y desarrolla en contextos sociopolfticos y no cabe su análisis al margen de la estructura de poder.

La historia de nuestros países ha sido un escenario de conflicto entre los poderosos $y$ los oprimidos, un proceso de dominación cuyas características y dureza han dependido de la correlación entre las fuerzas de opresión y la resistencia organizada del pueblo.

En la actualidad, en las llamadas "democracias" del Norte y del Sur, la globalización se ha convertido en una absurda carrera bacia el fondo, en que los países compiten, en realidad, por cual va a llegar primero a ser el peor en términos humanos para lograr la mayor tasa de explotación, tanto del ser humano, como de ia naturaleza. Los foros nacionales o internacionales que convoca el poder están secuestrados por los grandes monopolios y potencias, que los han convertido en grandes recintos de iegitimación y dominación; solo las asambleas y núcleos que han optado por una voluntad de emancipación social podrían ampliar los horizontes de la investigación y construir una actividad cientifica para la equidad.

La sociedad capitalista dejó hace mucho de ser el escenario de una clásica explotación de la fuerza de trabajo y de simple extracción de una tasa de plusvalia, mediada por condiciones básicas de seguridad laboral y bienestar social; ahora se trata de un sistema de dominio que ha degradado la subsistencia a lo minimo y ha creado la imposibilidad estructural para una reproducción social y ecológica sustentable.

Se ha dicho que en los anos sesenta la encrucijada se dio entre socialismo $y$ barbarie, una confrontación que se resolvió mediante la violenta imposición del terrorismo de Estado, orquestada desde Washington y la concertación estratégica de las oligarquias nacionales.(11) Ahora, tres décadas más tarde, la maquinaria neoliberal vuelve a provocar un cataclismo económico social y renueva la estrategia del terrorismo oficial, pero en esta oportunidad la encrucijada es mucho más violenta, pues se trata de la oposición entre pueblos que apenas sobreviven y una forma intensificada de barbarie capitalista. Todavia hasta mediados del siglo XX la legitimidad del sistema se consiguió mediante el hábil reconocimiento de algunos derechos humanos básicos y la negociación del llamado "pacto social", ahora el sistema ha demolido los derechos principales $y$ se sostiene fundamentalmente a base del miedo, el terrorismo institucionalizado en la guerra, y la expansión de los mecanismos de control cultural y hegemonia.

El mundo está sometido a la "coerción de una superpotencia que nos conduce a un mundo sin ley", como reza una carta reciente de Ramsey Clark - ex fiscal general de los Estados Unidos- al Secretario General de las NNUU. ${ }^{12}$ Las transnacionales han constituido una verdadera dictadura mundial, con un mando centralizado, aunque dependen de sus propios Estados. Un análisis recientemente publicado por la Revista "Fortune", sobre las cien trasnacionales más importantes, encontró que "...todas éstas se habían beneficiado de intervenciones específicas de los Estados nacionales donde tienen su base. El imperialismo está vivo $y$ los centros de poder monopólico no son virtuales y difusos, como pretenden hacernos pensar Negri y Hardt 13 ; tienen una organicidad y una correspondencia territorial; están enclavados en los aparatos de Estados concretos; se respaldan en ejércitos regulares o mercenarios que tienen fuentes de financiamiento $y$ centros de comando insertos en los servicios de inteligencia y los ejércitos del principal Estado imperialista; $y$ finalmente, la hegemonia y el control cultural también se ejercen desde maquinarias, medios de comunicación y aparatos culturales, cuyos centros de operación radican en dicho Estado imperialista.

¿Cómo se llegó a este retroceso profundo de los derechos humanos, sociales y culturales?

En un primer momento de la sociedad humana, existió un sujeto comunitario. Eran épocas en que los sujetos sociales operaban en función de valores de uso, que satisfacian necesidades establecidas por conveniencia colectiva: ni la división sexual del trabajo, ni las diferencias de género, ni los contrastes étnicos provocaron entonces desigualdades importantes; no porque se hayan tratado de sociedades ideales, sino porque el grado de desarrollo exigia equidad para la supervivencia.

Con la Conquista y la instauración de la sociedad colonial se quebró el sujeto comunitario, y surgieron sujetos privados guiados por el afán de atesoramiento mercantil; fueron rotos el derecho igualitario y la equidad, y junto con ellos se derrotó la concepción armónica del manejo de los bienes de la Naturaleza. La expropiación del oro y de la tierra, y la explotación feudal de la fuerza de trabajo fueron la 
base de la economia del mercantilismo colonial, provocándose la primera derrota de los derechos humanos y de la necesidad como eje de la construcción social.

En la entraria de esa primera etapa de la inequidad humana, se incubó una concentración de poder que reprodujo una triple jerarquización de la calidad de vida y del trabajo: la de pertenencia a una clase; la de pertenencia a una etnia; y la de pertenencia a un género. Triple inequidad social que para reproducirse requeria un Estado uninacional $y$ la uniculturalidad como forma de cultura oficial.

Ya entrada la época de nuestras repúblicas, sucederia la segunda gran derrota de los derechos humanos y de la necesidad como principio de definición social, cuando las mujeres y hombres pobres perdieron el derecho a la propiedad de los bienes fundamentales de la sociedad industrial. Aunque el llamado "sujeto obrero" logró - sobretodo en las sociedades industrializadas-, mantener con su lucha la vigencia de algunos derechos laborales, sociales y culturales básicos, aquel nuevo orden económico no permitió, con todo, que la política de pleno empleo se extendiese a las naciones periféricas y se difundiesen más equitativamente los beneficios.

Bajo la férula de relaciones neocolonialistas, esas naciones se tornaron exportadoras de productos primarios y se vieron impedidas de asumir a fondo la industrialización, con lo cual sólo un modesto porcentaje de su población conquistó los derechos humanos y sociales del antes mencionado Pacto de la Segunda Posguerra(14) . En esas condiciones se consolidó la subordinación no sólo de los trabajadores como clase, sino de los sujetos étnicos -indígena y afroamericano- y del sujeto femenino, mediante la instauración de un aparato educativo y políticas culturales eurocéntricas y androcéntricas.

Finalmente, desde mediados de los anos 80 se acentuó el carácter concentrador y monopólico de la economia y se abrió el nuevo período de la mercantilización global. La maquinaria neoliberal y

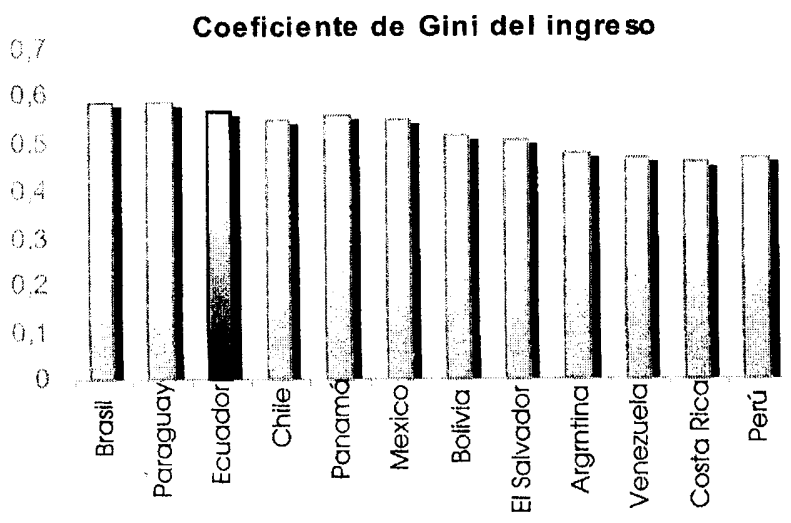

sus mecanismos de recomposición productiva provocaron un cataclismo económico social, y para sostenerse renovaron la estrategia del terrorismo oficial; sólo que ahora la opresión no se presenta en la forma directa de las dictaduras, sino que se ejerce a través de la administración del miedo y la inseguridad como forma de gobierno. Comenzó así la tercera y más profunda derrota de los derechos humanos y una polarización sin atenuantes de la sociedad. Es un modelo social perverso, porque aunque teóricamente se esgrime como una salida a los errores del keynesianismo y como un proyecto para colocar la riqueza acumulada por las empresas al servicio de la gente, lo que en verdad se ha montado es una maquinaria de demolición de los derechos, un mecanismo institucional y jurídico para imponer politicas de minimización de la cesta familiar a niveles de hambre e institucionalizar la apropiación fraudulenta de los recursos estratégicos, de los fondos de ahorro y jubilaciones de los ciudadanos. Al haberse constituido ahora más que nunca la vida humana alrededor del afán productivo, y la producción alrededor del sediento cálculo de intereses de los monopolios privados, las actividades productivas menosprecian los impactos de tal actividad sobre la vida (humanidad y naturaleza), de ese modo el crecimiento económico está impedido de ser una via de desarrollo humano. Por el contrario, desde el punto de vista epidemiológico, mientras las clases dominantes, a pesar de su fracaso siguen esgrimiendo la privatización como la panacea de todos los males, lo único que se sucede es que se privatiza la riqueza mientras se socializan el hambre $y$ las epidemias. En una calle de Quito, aparece un graffiti que pinta de cuerpo entero el engano: "i Si las privatizaciones son el remedio, porqué Argentina agoniza?"

Gracias a la imposición de un régimen de salarios por debajo del precio de la fuerza de trabajo, a esa estructura monopólica que expulsa a la masa de subempleados y desocupados a los círculos secundarios de la economia, para 1998 el Brasil $y$ mi país el Ecuador pasaron a ser los países con mayor desigualdad en el ingreso. Tanto como se comprueba al observar el elevado índice de Gini para la concentración del ingreso. ${ }^{(15)}$

E1 caso del Brasil es muy ilustrativo, porque siendo el país más rico de América del Sur ostenta los contrastes dramáticos entre su alto índice de concentración de la riqueza, el mayor de América, con los pésimos indicadores sociales y epidemiológicos, que lo colocan, también en esa faceta dei sufrimiento humano, a la cabeza de la región.

Los conceptos que se han utilizado para caracterizar la situación de salud en América Latina y el Tercer Mundo son las de catástrofe u holocausto, para significar la magnitud del impacto del capitalismo 
posindustrial en la salud, no tiene sentido volver aqui con cifras latinoamericanas de la debacle epidemiológica, ampliamente conocidas en los círculos de la salud pública. Es en alusión a este tipo de problemas que Castiel 16 nos recuerda una definición de Epidemiología como la "teoria de la catástrofe en cámara lenta".

Tenemos por consiguiente frente a nosotros, el escándalo de un mundo en destrucción, el crecimiento irrefrenable de la inequidad, la ampliación de la brecha entre la riqueza acumulada por los grandes monopolios y las clases en el poder y los niveles infrahumanos de supervivencia de la inmensa mayoría, y ante todo eso, la presencia de una capa de intelectuales dorados que le hacen el juego al sistema, con su discurso tecnocrático, con sus investigaciones expiatorias, y que con sus aportes intelectuales e información, contribuyen indirectamente a mantener a un sector de las masas sin brújula ("bússola") o secuestrado en reivindicaciones moralistas, o demandas inofensivas para un mejor nivel de consumo, en lugar de contribuir al desarrollo de su conciencia profunda sobre la estructura de dominación que se ha globalizado, pero que en cada Estado nacional se reproduce sin piedad, provocando una regresión de los derechos sociales y políticos, ligada al sistema de propiedad y distribución social. ¿Cómo creer en el discurso de la equidad y de salud para todos que emana de los círculos del poder, y que propugnan algunas agencias de cooperación y Naciones Unidas, sin mirar, por ejemplo, la amenaza dei modelo de integración que se nos quiere imponer con el nombre de ALCA, y que esta diseñado exactamente para reproducir y ampliar la profunda inequidad regional a nombre de la libertad de comercio? Es por eso que se hace urgente apoyar la construcción de sujetos históricos capaces de pensar criticamente y transformar la realidad; un desafio que se proyecta hacia la necesidad de transformar el propio sujeto de la investigación.

\section{Salarios reales en Latinoamérica} 1980-96

(Indice 1987=100)

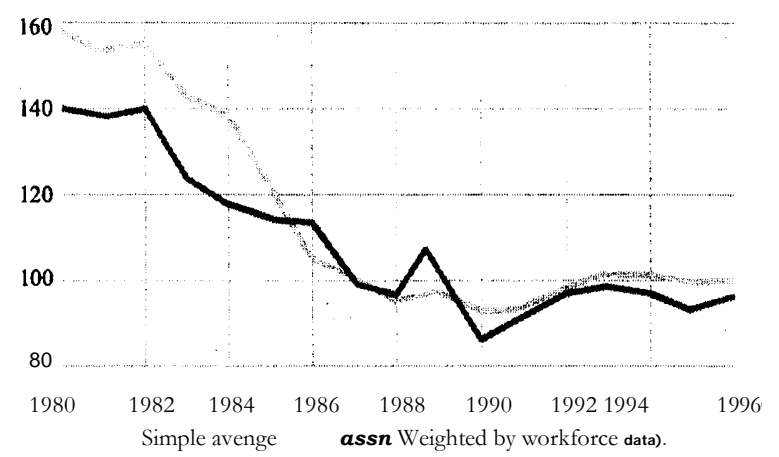

\section{TRAYECTORIA ESPACIO TEMPORAL DEL SUJETO: EL IMPERIO DE LA UNICULTURALIDAD}

En la primera parte de este escrito, mencionamos el proceso histórico por medio del cual se crearon condiciones para la implantación de una visión unicultural en la ciencia dominante: primero la violenta instauración, en la Colonia, de un Estado uni-nacional, que institucionalizó la uniculturalidad en el aparato oficial y coartó la participación de los sujetos indígena, afroamericano y femenino-; luego los anos de formación de la época republicana en que se consolidaron el modernismo positivista con su eurocentrismo y el androcentrismo en el aparato cultural y educativo; hasta llegar en décadas recientes al posmodernismo conservador como una fase histórica de atomización radical del sujeto social y la reinstauración de una ideologia individualista, expresada en vertientes tan distintas como el ultraliberalismo y el relativismo cultural. En última instancia, sea cuando ese individualismo operó bajo la forma tipicamente Occidental, o aun cuando se vistió de un supuesto multiculturalismo, lo que está implicado es la absorción final de todas las culturas en una cultura global y acrítica, que puede estar dispuesta a reconocer el pensamiento y las expresiones culturales de los otros sujetos, siempre y cuando no se mantengan referentes colectivos emancipadores y se respete la hegemonia de la cultura dominante. No podemos detenernos aqui en una explicación de cuales fueron las mediaciones que, como la lengua, permitieron la penetración de dicha uniculturalidad, lo que interesa resaltar es que esa tendencia no sólo influyó la cultura latinoamericana general, sino aun la formación y reproducción de nuestro pensamiento científico unicultural, convertido así, directa o indirectamente, en un instrumento de reproducción de una estructura de poder, atravesada por relaciones de apropiación y expropiación económica, como por relaciones de subordinación étnica y por relaciones inequitativas de género.

Claro está, esa dominación $\mathbf{y}$ hegemonia no implicaron nunca la anulación total de los sujetos dominados, que han permanecido activos y en lucha históricamente, y que ahora reclaman un espacio para su voz, pero si implicó que los conceptos, métodos e instrumentos predominantes en nuestro quehacer mantuvieran por muchos anos, como único referente el del pensamiento "Occidental".

De cara al desafio de construir un nuevo paradigma, dicha uniculturalidad aparece ahora como un importante obstáculo para un ciencia emancipadora, y su superación depende de la capacidad que tengamos para implementar un trabajo cientifico intercultural. Es necesario integrar los conocimientos 
emancipadores provenientes de distintas fuentes del saber: el conocimiento académico; la ciencia de base ancestral de los pueblos ["ciencia de lo concreto" en el sentido planteado por Levi-Strauss]; e incluso el saber común sistematizado por las colectividades urbanas y rurales; y extraer de ese acumulado lo necesario para construir objetos/conceptos/campos de acción contrahegemónicos, discriminando aquellos elementos culturales que nos atan a un pasado de subordinación y que restringen nuestra acción a lo formal y funcional.

Lo dicho implica convocar otros actores al trabajo de construcción sobre el saber en salud, argumento polémico que nos exige demostrar hasta que punto es factible tal integración, y comprobar que una propuesta de esa magnitud, no implica un radicalismo que desconoce la necesidad de una praxis científica especializada.

Reconozcamos para empezar, que no se trata exclusivamente de que el sujeto académico absorba y recree los documentos $y$ evidencias provenientes de otras formas de producción de conocimiento, como parte de ese paso "normal" de recolección bibliográfica y recopilación documental; es más bien un desafio para perfeccionar un proceso realmente intercultural que permita, como diria Walsh, la traducción reciproca de conocimientos, la construcción conjunta del objeto de transformación y del marco interpretativo, la aparición de nuevas formas de análisis y la aparición de conceptos renovados; implicaria la creación de un marco epistemológico integrador que ampare la "negociación" de conocimientos, una nueva estructuración, y un [in]disciplinamiento de ias estructuras que se integran para que se atienda a los significados y especificidades de las zonas de contacto" (17). Y nosotros añadiríamos, para que se vigorice la capacidad crítico analítica y se renueven los paradigmas de acción, desde una perspectiva realmente emancipadora; prueba de fuego de una propuesta intercultural que quiera aportar a un verdadero nuevo proyecto para la salud.

Ese paso significa la conjunción del poder explicativo de diversas formas o expresiones del pensamiento, pero no de cualquier pensamiento sino del pensamiento crítico. Un movimiento que avanza interculturalmente hacia una metacrítica de la sociedad capitalista, que no es la simple sumatoria de las capacidades explicativas y del poder transformador de los grupos diversos, sino que implica una dimensión nueva, una superación dialéctica.

Pero, tenemos que asegurarnos que dicha apertura multilateral del pensamiento crítico no nos )leve a una diversificación absoluta, sin un concomitante movimiento de unidad; pero ya no la unidad dominada por una sola cultura, como tampoco la unidad que se construye alrededor de ningún "relato matriz" o "narrativa maestra" que se impone sobre las otras, sino la construcción de un relato unificador o metadiscurso, que sea unificador sin ser dominador, y que pueda orientar discurso emancipadores complementarios. Un metadiscurso en el que confluyan todas las expresiones emancipadoras del pensamiento crítico de la Modernidad. En ese sentido, la mirada en ese filosofia del futuro, no puede llevarnos a la exclusión dogmática y tendenciosa de ningún pensamiento emancipador, peor aun de aquellos que han constituido el discurso y la práctica de los pobres, de los dominados y de los sin poder. Para que las ciencia contemporáneo gane un puesto en la lucha por la humanidad, no sólo tiene que velar por la rigurosidad de sus procedimientos y el avance de sus técnicas, sino que tiene que articularse a la construcción de esas nuevas meta narrativas de la liberación.

La construcción emancipadora intercultural del conocimiento seria entonces una superación dialéctica de la construcción emancipadora convencional de la izquierda mestiza, que ha sido en gran medida monocultural, no sólo en el sentido de su línea eurocéntrica, fuertemente referenciada por los parámetros del pensamiento de la Modernidad "Occidental", sino también monocultura) en su forma de ser una ciencia centrada en la visión masculina.

\section{ALGUNAS CLAVES PARA UNA INVESTIGACIÓN RESPONSABLE}

Nos resta exponer algunas claves que a nuestro criterio, podrian orientar el debate sobre como caminar hacia esa meta narrativa y la construcción de nuestras tareas futuras en la investigación, que aqui apenas podemos bosquejarias.

\section{El Cambio de la Noción de lo Que es Práctico: Investigación Reformista o Instrumento de Contrahegemonia}

Es evidente que la vida nos reclama una investigación con los pies en la tierra, pero eso puede significar visiones muy distintas. Para unos, es la instrumentación inmediata de medidas posibles dentro de los limites de la estructura de poder vigente, es decir dentro de las regias del juego del capitalismo, para otros, es la instauración de un proceso de construcción contrahegemónica, sustentable y conducida por ias organizaciones politicas y sociales del pueblo, hacia una nueva forma de sociedad. La comprensión de esa diferencias tiene que ver con la distinción entre reformismo, como política del cambio de formas que no alteran la sustancia de la estructura de poder, está ligada a una concepción funcionalista 
de la gestión y a una filosofia liberal, y emancipación que implica la creación de formas alternativas, que compiten y superan elementos de dicha estructura de poder para crear nuevos modos de vida, que puede pasar por momentos estratégicos de reforma, pero que está ligada a un proceso social de construcción de contrahegemonpia y a una filosofia de la emancipación.

No se trata aqui de volver al lysenkismo, ni abogar por una instrumentalización barata del pensamiento científico, el cual como el arte, requiere de un margen de autonomia relativa para su pleno desarrollo; no se trata, en nuestro caso, de teñir de ideologia a ias células, los neurotransmisores y los músculos; se trata simplemente de superar ias restricciones del pensamiento científico convencional, que está presente en la mayor parte de artículos $y$ escritos que circulan en el "mainstream" de la bibliografia.

Si estamos dispuestos a ser prácticos en un sentido emancipador, tenemos que estar dispuestos también a un trabajo teórico. La teoria vale, y debemos abrir puentes entre los escenarios académicos y técnicos, sin caer en el falso dilema de si los conocimientos teóricos deben servir para desentravar la lógica histórica del sistema capitalista, o si por el contrario deben servir como prescripciones para la labor práctica. La teoria se aplica tanto para la comprensión de la estructura de reproducción del capitalismo, cuanto para entender los procesos generativos y el movimiento d ela participación y organización colectiva, debemos conectar ambos para evitar una visión reduccionista de la investigación y la politica.

\section{La Distinción de Inequidad y Desigualdad}

La confusión que impera actualmente sobre el concepto inequidad, no sólo es un obstáculo para el avance de una investigación verdaderamente ligada a la emancipación en la salud colectiva, sino que nos permite ilustrar como la falta de claridad puede llevarnos a una proyección funcionalista.

Para impulsar la construcción de un mundo distinto no es suficiente con describir las profundas desigualdades sociales y de salud, sino que es indispensable poner al descubierto las raices de esas desigualdades. La inequidad es la falta de equidad que surge como producto de la concentración de poder en unas clases sociales, o en ciertos grupos étnicos o en uno de los géneros; es decir, es la característicainherente a una sociedad que impide el bien común, e instituye la inviabilidad de un reparto humano que otorgue a cada quien según su necesidad y le permita aportar plenamente según su capacidad; por eso el estudio de la inequidad es un punto crucial para el análisis de los derechos humanos. La desigualdad, en cambio, es una expresión observable tipica y grupal de la inequidad; expresa un contraste - de una característica o medida- producida por la inequidad.

Si nos quedamos en el nivel de la desigualdad, estariamos reduciendo o desviando nuestra mirada a los efectos que se provocan, en lugar de enfocar sus determinantes.

Partimos entonces de reconocer que hay marcados contrastes en el disfrute de los derechos humanos en ias sociedades, y que corresponden a las relaciones de poder que caracterizan y separan los grupos sociales con sus modos de vida, de los cuales forma una parte decisiva el disfrute de aquellos derechos. Igualmente, en el seno de dichos modos de vida grupales ocurren estilos de vida singulares o individuales *. Las relaciones de poder son las que discriminan los grandes contrastes entre los modos y estilos de vida de grupos situados en los poios sociales de una sociedad, así como la capacidad de producción $y$ negociación que los grupos tienen para la reproducción de su vida en condiciones determinadas. La estructura de poder está dada por la relaciones entre clases sociales, géneros y grupos étnicos que se enfrentan porque la concentración de unos afecta la exclusión de otros respecto al disfrute de los bienes y riqueza de una sociedad. Esta es una condición determinante de la salud y su reparto.

\section{De la Etica de la Práctica a 'la Etica del Modo de Vida}

Cuando rebasamos un óptica exclusivamente médico-asistencial, comprendemos que la salud humana no depende solamente de los servicios de salud, sino que se moldea en todos aquellos otros espacios o escenarios, que contribuyen poderosamente para el estado de salud, tanto de las personas y sus familias como de los grupos sociales a los que pertenecen; el trabajo; la vida del consumidor; la vida cultural; la vida de organización $y$ soportes colectivos; y las relaciones ecológicas.

Lo anterior nos obliga a ampliar el paradigma bioético e introducir en el campo del debate sobre bioética, el de las condiciones que afectan notablemente

\footnotetext{
* Vieira mira estas relaciones desde el punto de Bourdie que las enfoca más bien como interacciones de los individuos, en medio de una triada "espacio social - campo - habitua" y observa las relaciones entre las posiciones de loa individuos en el espacio social, las interacciones con otros individuos que participan en un mismo campo de interacciones de sus estilos de vida, que serian producto de su habitus. El habitus seria un principio generador de prácticas objetivamente reconocibles y a la vez un sistema de segregación de dichas prácticas, y sobre esas dos características es que se constituiria el mundo social representado, es decir el espacio de los estilos de vida (Vieira 1999).
} 
el campo de la salud poblacional y de nuestros pacientes, por ejemplo: el racismo, las desigualdades de género, el hambre, la miseria, la diseminación de drogas, las situaciones de abandono infantil y de los ancianos; la dimensión ética en la salud de la colectividad como lo dirian Berlinguer y Garrafa. Y para incorporar estas preocupaciones la bioética tiene que preocuparse de problemas como: derechos humanos, ciudadania, libertad, participación, autonomia, igualdad y complejidad, equidad, calidad y excelencia, radicalidad $\mathrm{y}$ tolerancia. Asuntos todos estos que no se hacen visibles ne la investigación convencional, ni alcanzan mayor importancia cuando la bioética se circunscribe a los temas que atañen la práctica del consultorio.

\section{De la Investigación Participativa a la Producción Intercultural del Saber:}

\section{De la Gerencia del Conocimiento a la Planeación del Saber}

Los avances de las ciencias administrativas aplicadas al desarrollo y las nuevas teorias sobre la gestión-y la gerencia como una de sus herramientas-, han determinado la creciente necesidad de discutir las articulaciones entre el esfuerzo social y la gerencia.

El conocimiento es una herramienta social $y$ como todo otro recurso de la vida está sometido a las contradicciones $y$ presiones que chocan en una sociedad clasista e inequitativa. La planeación de una investigación alternativa, no puede desentenderse de esa conflictividad y tiene que diseñarse bajo plena conciencia de las presiones contrarias que operan en la actualidad sobre el conocimiento: al mismo tiempo que importantes núcleos trabajan para elaborar propuestas innovadoras de la teoria de la administración del conocimiento, así también se han desencadenado desde la orilla conservadora de la sociedad propuestas para la gestión y la gerencia que son ciarísima parte de la hegemonia.

El cambio hacia la sociedad posindustrial de mercado, con su recomposición del sistema económico hacia redes virtuales de empresas, viabilizado por el flujo y disponibilidad instantánea de información, sobre la base técnica de los recursos de la comunicación digital, la teleinformática y los hipermedia, es lo que algunos han descrito como la economia del conocimiento ("knowledge economy") o la sociedad basada en el conocimiento ("knowledge based society"). En dicho escenario del mercado instantáneo y de la administración de empresas globalizadas en tiempo real, los temas centrales de la lucha económica son la flexibilidad y la adaptabilidad para viabilizar la competitividad. Por eso es que la "gerencia del conocimiento" tuvo que surgir en el seno de la preocupación de las empresas modernas, o mejor dicho "posmodernas" para asegurar flujos y procesos, y para aprovechar cualquier elemento del conocimiento con miras a un proceso productivo más ágil.

Nosotros tenemos que preguntarnos si lo que queremos es un conocimiento que agilice la funcionalidad, o contribuya a empoderar a los ciudadanos de las bases para construir una contrahegemonía en salud.

Ahora bien, el movimiento del conocimiento en una colectividad tiene varios momentos y espacios de realización. Desde el punto de vista que aqui nos ocupa, interesa destacar que la planeación del conocimiento incluye tanto la producción de conocimiento, cuanto la gestión del mismo. La producción del conocimiento a su vez involucra tanto el momento de generación del conocimiento (es el movimiento de génesis de productos del conocimiento, que incluye la elaboración de conceptos y la realización de un método, mediante la formulación de descripciones y explicaciones en un escenario práctico), cuanto el de reproducción del conocimiento. $\mathrm{Si}$ buscamos la generación y reproducción intercultural del conocimiento tenemos que implementar espacios y mecanismos para enlazar la gestión del conocimiento institucional con la gestión colectiva del saber.

Por otro lado, una gestión en la converjan un pensamiento crítico sobre la gestión y una población organizada e informada adquieren un poder decisivo para transformar los problemas y para mantener un proceso de avance del conocimiento y del saber. Y la planeación del saber juega un papel clave tanto en la construcción de dicho pensamiento crítico, cuanto en el impulso de una participación informada.

Las relaciones de dominación y explotación que caracterizan a nuestra sociedad para reproducirse de un modo duradero requieren que las clases dominadas acepten una especie de intercambio de servicios con las clases de arriba; la hegemonia requiere que las clases dominantes penetren las instituciones, los objetos y los mensajes no sólo sus propios intereses sino parcelas de las culturas e interese populares que son aceptadas como Atiles por la mayoría. La investigación funcionalista alimenta ese tipo de maniobra.

Una gestión colectiva contrahegemónica sólo puede realizarse básicamente a través de tres mecanismos: la planeación estratégica; el monitoreo participativo de la colectividad sobre la salud y sus elementos; y el control social sobre la gestión. En todos esos momentos se requiere con urgencia una investigación alternativa. 


\section{La Investigación y el Neohumanismo Popular}

Un proyecto alternativo para la investigación latinoamericana presupone que los/las trabajadores/ as de la ciencia nos reconozcamos como sujetos nuevos -el nuevo ser humano que soñó el Che-, situados en la globalidad de la vida de reproducción colectiva, que forma parte de la reproducción de la naturaleza. Esa característica humana de reinventarnos permanentemente, de ser críticos y de recrear la vida, se liga también a nuestra profunda tendencia a compadecermos por todos y por todo, sin egoísmos, $y$ siempre sovando con un mundo solidario, emancipado de toda forma de concentración del poder. Es decir, necesitamos reafirmamos precisamente en lo contrario de lo que persiguen quienes quieren dominarmos, convirtiéndonos a los investigadores en tecnócratas competitivos y sumisos al poder. Eso es lo que hemos denominado neohumanismo popular. Las tesis humanistas siempre estuvieron ligadas a las formas de ver y construir la cultura de los grupos emergentes, de los sin poder. El neohumanismo se recrea en los proyectos que luchan contra la inequidad, que buscam humanizar el modo de vida; se recrea en los municipios donde existen autoridades del pueblo; se reafirma en los proyectos de economia popular paralela; en las asambleas de barrios, de piqueteros; se recrea en la lucha de los indigenas; en las conquistas de género; en definitiva, en todos esos espacios donde confluyen, como diria Gramsci, el movimiento organizado de las masas, pero atravesado por el pensamiento crítico, y el pensamiento crítico de los expertos, pero atravesado por la vida y las necesidades de las masas. ${ }^{(18)}$

Nuestra concepción neohumanista, que nos reafirma como fabricantes de utopias, va de la mano con nuestra visión solidaria con los otros y las otras, con nuestra gente, con quienes mantienen con vida la riqueza de nuestras culturas, en un proceso de permanente renovación. Por eso debemos buscar una ciencia que nos permita afirmarmos como sujetos, sabiendo que nuestra propia afirmación depende de la afirmación de los otros sujetos, y también presupone de la afirmación de la naturaleza.

La ciencia debe trabajar para integrar los conocimientos y expresiones progresistas de las clases subordinadas, de los pueblos y nacionalidades postergadas y de los géneros subalternos, integrándose a la construcción de un bloque popular que facilite la construcción intercultural del saber. La democratización profunda de la sociedad requiere de un proceso simultáneo de democratización y descentralización del Estado, que se construye bajo interrelación con un proceso de construcción de poder económico, político y cultural de la sociedad civil. No estamos aqui pensando en esa versión inocua de sociedad civil, sino en la sociedad civil de los pobres.(19)
Como lo dijéramos en nuestro libro de "Epidemiologia Crítica" tenemos que trabajar por defender aquello que es la base de una salud verdadera: "...La seguridad humana integral; la triple equidad de acceso y participación -social, étnica y de género-; la conquista del derecho universal a servicios y programas de la más alta calidad; el impulso urgente de un proceso de humanización y protección de la vida en todas sus dimensiones -laboral, del consumo, de la reproducción cultural y subjetiva, de la promoción y defensa de una ecologia saludable y de la implementación de una construcción multicultural de las formas y sentidos de la organización-. Todos estos son puntos nodales de la nueva política por la que luchó el movimiento de la medicina social latinoamericana desde su nacimiento en los años $70 \mathrm{y}$ desde su aparición formal en el Congreso de Ouro Preto en 1985, y que ahora gracias a la alquimia de unos cuantos tecnócratas obsecuentes se han borrado de las agendas o han sido trasmutados en ellas a simples elementos de una confusa retórica neofuncionalista."

Atravesados por un período histórico en el que los dueños de los monopolios han sido eficaces para organizar un sistema más agresivo de acumulación, que, como lo dijimos antes, dejó de ser una simple explotación de la fuerza de trabajo y extracción de plusvalía medida por condiciones mínimas de seguridad humana y sustentabilidad del planeta, para convertirse en un sistema implacable basado en la degradación máxima de la población, en el asalto a los derechos humanos y en la explotación ciega de los recursos de la Tierra, de esta forma se ha llegado a la imposibilidad estructural de una reproducción social sustentable. La investigación no puede ponerse al servicio de ese apetito irrefrenable, ni de modo directo, como tampoco puede hacerse la desentendida sobre la maquinaria de destrucción humana que se ha montado, con tal de que le permitan mantener sus espacios de realización.

Pero no debemos desmayar en nuestro Animo, porque las fases conservadoras del Estado como la que atravesamos no son necesariamente épocas de estancamiento y anulación del espíritu innovador, pueden ser períodos creativos, de preparación y acumulación de una saber, de experiencias y organización contrahegemónicas.

Vamos a retomar el hilo conductor del pensamiento crítico para realizar una investigación que vibre con los mismos suemos, creaciones, alegrias y dolor, que hacen parte de la belleza indomable de nuestros pueblos. 


\section{REFERÊNCIAS BIBLIOGRÁFICAS}

(1) Breilh J. (2002). Epidemiologia crítica: hacia un paradigma emancipador e intercultural de la ciencia y el conocimiento". Buenos Aires: Lugar Editorial (2002)

(2) Morin E. Ciência com conciencia. Rio de Janeiro: Bertrand Brasil; 1996.

(3) Santos B. Introdução a uma ciência pos-moderna. Porto: Ediciones Afrontamento; 1995

(4) Latour B. Pandora's Hope (Essays on the Reality of Science Studies). Cambridge: Harvard University Press.; 1999

(5) McLaren P. Multiculturalismo crítico. São Paulo: Cortez; 1997

(6) Walsh C, Schiwy F, Castro-Gómez S. Indisciplinar las ciencias sociales. Quito: Universidad Andina Simón BolivarEdiciones Abya-Yala.; 2002

(7) McLaren P. Multiculturalismo Crítico. Sao Paulo: Cortez Editora. 1997

(8) Kuhn, T. The Structure of Scientific Revolutions. Chicago: University of Chicago Press; 1962

(9) Almeida Filho N. La ciencia tímida. Buenos Aires: Lugar Editorial; 2000

(10) Breilh J. Epidemiología critica: hacia un paradigma emancipador e intercultural de la ciencia y el conocimiento. Buenos Aires: Lugar Editorial; 2002

(11) Dieterich Steffan H. Hacia el cataclismo latinoamericano en "Cartas al Libertador" <rcm@andinanet.net: 60, febrero $17>$

(12) Clark R. Carta Aeceratrio General de Naciones Unidas. Trad de Loles Oliván CSCA (online) new york: Naciones Unidas <www.nodo50/org/csca> (20 sept 2002)
Hardt M, Negri A. Imperio. Trad. de Eduardo Sather. Massachussets: Harvard University Press; 2000

(14)Betto F. Economia y ciudadania. Sao Paulo: Servicio Informativo de Alai-amlatina, 2002

(15) BID. Progreso Económico y Social en América Latina: Informe 1998-99. Washington: 1999

(16) Castiel L. Apocalypse...Now? Molecular epidemiology, predictive genetic tests, and social communication of genetic contents. Cadernos de Saúde Pública 1999; 15(Supl 1): $73-89$

(17) Walsh C. ¿Qué Conocimientos? Reflexiones sobre las Politicas de Conocimiento, el Campo Académico, $\mathrm{y}$ el Movimiento Indigena Ecuatoriano. Boletín ICCI 2001; 25

(18) Kanoussi D. Una Introducción a los Cuadernos de la Cárcel. Puebla: Plaza y Valdez; 2000

(19) Hidalgo F. Alternativas al neoliberalismo y bloque popular. Cuenca: CINDES / U. de Cuenca /CETRI; 2000

$(13)$ 